\title{
ANÁLISIS TERMINOLÓGICO DEL MANUAL GEOMÉTRICO (MADRID, 1640) DE JUAN CARLOS DELLA FAILLE*
}

\section{TERMINOLOGICAL ANALYSIS OF JAN-KAREL DELLA FAILLE'S GEOMETRIC MANUAL (MADRID, 1640)}

\author{
Francisco Javier Sánchez Martín \\ Universidad de Murcia \\ javisanmar@um.es
}

\section{RESUMEN}

En el marco del corpus científico-técnico constituido para el proyecto de investigación enfocado al estudio de la lengua matemática española del siglo XVII, cabe destacar el manuscrito Método de la geometría (Madrid, 1640), fruto de la actividad científica y docente del matemático Juan Carlos della Faille en el Colegio Imperial madrileño.

Si bien el tecnolecto matemático, en coincidencia con otros ámbitos de especialidad, presenta características morfológicas y sintácticas prototípicas, además de modelos textuales y discursivos específicos, sus particularidades lingüísticas residen primordialmente en el vocabulario técnico, ámbito preferente de atención en este trabajo.

El análisis del plano léxico prestará atención a diferentes aspectos, tales como significados, transmisión y documentación. Para ello partimos de los datos y de la información que nos proporciona su autor para someterlos a análisis y compararlos con los que nos ofrecen otras fuentes (corpus en línea, obras históricas o diccionarios). Así, Juan Carlos della Faille declara en su obra la importancia de la claridad y la clasificación conceptuales, en paralelo con la destreza en el estudio de la perspectiva y su aplicación práctica en la delineación objetual.

Por otro lado, a partir de numerosos ejemplos, se pondrá de manifiesto que las imágenes han guiado, al lado de las palabras, nuestra comprensión; dicho apoyo visual permite, en ámbitos como el geométrico, descifrar la formulación de problemas planteados por los matemáticos.

Palabras clave: lexicología, neología, matemáticas, Juan Carlos della Faille, siglo XVII

\begin{abstract}
Within the technical-scientific corpus made for this research project that focuses on the study of the 17th century Spanish mathematical language, it is noteworthy the manuscript 'Método de la geometría' (Madrid, 1640), result of the scientific activity and teaching of the mathematician Jan-Karel della Faille in the Imperial College Madrid. While the mathematical technolect, in conjunction with other areas of the field, presents prototypical morphological and syntactic characteristics along with textual and discursive models, its linguistic characteristics mainly lie in the technical vocabulary, which is the focus of the present study.

The analysis of the lexical component will focus on different aspects like meaning, transmission and documentation. We start from the data and information given by the author, and we analyse and compare it with those offered by other sources (online corpus, historical works or dictionaries). In this way, Jan-Karel della Faille states in his work the importance of clarity and conceptual classification, in parallel with the ability in the study of perspective and its practical implementation in object delineation.

On the other hand, from numerous examples, we will highlight the use of images, together with words, that led our understanding. This visual aid allows us to decipher the formulation of problems posed by mathematicians in areas such as geometry.
\end{abstract}

Keywords: lexicology, neology, mathematics, Jan-Karel della Faille, 17 th century

\footnotetext{
* Este trabajo es resultado del proyecto Corpus para el estudio de la lengua española científica y matemática del siglo XVII (19296/PI/14), financiado por la Agencia de Ciencia y Tecnología de la Región de Murcia.
} 


\section{MOTIVACIÓN PARA EL ESTUDIO DE A OBRA DE JUAN CARLOS DELLA FAILLE}

El matemático y preceptor de corte Juan Carlos della Faille ${ }^{1}$ (Amberes, 1597Barcelona, 1652) -descendiente de una familia de prósperos comerciantes flamencosestudió Humanidades en el colegio de Amberes (1609) antes de entrar en la Compañía de Jesús $(1613)^{2}$. Este jesuita matemático está en la estela de los grandes matemáticos contemporáneos (Meskens 2005). En sustitución de Saint-Vincent, dada la imposibilidad de este para asumir el puesto por su enfermedad, Juan Carlos della Faille fue enviado a Madrid, el 23 de marzo de $1629^{3}$, para ocupar una de las cátedras de matemáticas que se habían establecido en los Reales Estudios del Colegio Imperial, institución fundada el 23 de enero de $1625^{4}$ (Van der Vyver, 1977, p. 77, López Piñero, 1983, s.v. La Faille, Jean Charles de).

Cabe mencionar la relevancia del trabajo matemático del antuerpiense, especialmente en relación con sus escritos en latín sobre las secciones cónicas (e incluso pese a que, desafortunadamente, sus escritos sobre esta temática no vieron la luz) para los que usa conceptos de la geometría proyectiva, aunque nunca los define como conceptos especiales al llegar a los mismos - como es obvio- desde el marco de la geometría clásica (vid. Meskens, 2005, p. 104-105).

De los fondos de los manuscritos científicos conservados por la Real Academia de la Historia procede el presente manuscrito objeto de edición y estudio, Método de la

\footnotetext{
${ }_{1}^{1}$ Adoptamos el nombre empleado en la mayoría de los tratados escritos por él en nuestra lengua frente a otros antropónimos empleados en lenguas foráneas, según constata el estudio de Meskens (2005, p 6): "Jean Charles is used in contemporary French literature, while Jan-Karel is usual in Dutch literature. He is refered to as Juan Carlos in several of the treatises he wrote in Spanish. In most of his correspondence, however, he used the name he was baptized with, Joannes".

2 "That he became a Jesuit is no surprise, seeing that many sons of wealthy merchants entered a religious order in what was a 'frontline' town of the Counter-Reformation" (Meskens, 2005, p. 22).

${ }^{3}$ En Meskens (2005, p. 44-46) puede leerse otra diferente versión sobre el destino a nuestro país de Juan Carlos della Faille como segundo profesor de matemáticas al lado de su maestro. La primera cátedra de matemáticas fue impartida por el padre Claudio Ricardo desde septiembre de 1629.

4 También llamado durante esta etapa (1625-1767) impropiamente por algunas monografías Colegio de San Isidro. Con anterioridad cabe destacar la labor cumplida por la Academia Real Matemática fundada por Felipe II en 1582, de cuyo estudio se ocupa Sánchez Martín (2009, p. 37-46). Por su parte, Durán Guardeño (2000: 233) se muestra partidario de sostener la nula influencia de dicha academia, a la vez que considera que uno de los principales círculos matemáticos europeos de la primera mitad del siglo XVII fue la Accademia dei Lincei (Academia de los linces) fundada en Roma (1601) y a la que perteneció Galileo. Tras su desaparición treinta años después, vino a sustituirla la Accademia del Cimento, cuya fundación formal en Florencia sucedió en 1657, pese a su funcionamiento y germen una década antes.
} 
geometría (1640), obra citada en el estudio sobre el Colegio Imperial realizado por Simón Díaz (1992), pero que hasta la fecha estaba sin editar.

El manuscrito de Juan Carlos de la Faille supone una muestra de la actividad científica producida en esta etapa del devenir histórico, y con la que plasma el estado y el grado de difusión de los conocimientos en tanto que factor determinante para el progreso de la ciencia.

En cuanto a la parcela geométrica, la edición de estos manuscritos inéditos del siglo XVII, realizada en el marco del proyecto dedicado al estudio histórico de la lengua matemática española, supone una muestra del material textual hispánico que pretende servir de instrumento para el análisis sobre la evolución histórica del léxico científico español en este ámbito temático y en un periodo cronológico poco atendidos.

De hecho, cada vez con más frecuencia, los especialistas en la historia del lenguaje científico vienen dirigiendo su mirada a un mayor número de ámbitos temáticos y de periodos cronológicos, lo que está posibilitando reconstruir la historia del léxico científico. Su estudio, por lo tanto, “debiera tener reservado un lugar fundamental entre las tareas de la historia de la ciencia y la de la lengua”-sostiene Gutiérrez Rodilla (2016, p. 117)-, ya que permite comprender la historia del propio discurso científico y, a la vez, completar la historia misma del léxico de nuestro idioma. Esta prosa científica constituye la manifestación directa de su expresión especializada, de ahí que resulte ineludible partir de su testimonio para acometer su estudio filológico y lingüístico.

\section{APORTACIONES DE LA OBRA PARA LA HISTORIA DEL LÉXICO MATTEMÁTICO}

Es conocido el gran desarrollo que el español experimentó durante el Siglo de Oro en la parcela del vocabulario científico, renovación lograda bien por medio de los mecanismos endógenos al sistema lingüístico de nuestra lengua bien merced a la entrada de cultismos o de los neologismos de préstamo. En el ámbito del análisis de las características de los niveles lingüísticos, el léxico resulta el rasgo más prototípico del Método de Geometría, en tanto que obra constitutiva del corpus de carácter técnico reunido, como se expone en Sánchez Martín (2018), no es extraño el hecho de que una de las particularidades de las lenguas de especialidad resida fundamentalmente en la 
utilización del vocabulario técnico apropiado a las circunstancias comunicativas. Además, como es consabido, entre el léxico de un idioma y los conceptos estructurados de la ciencia se entabla una relación de necesidad. Por consiguiente, un ámbito particular de la historia de la ciencia y la de la lengua es la reconstrucción de la historia del léxico científico, una tarea insoslayable, según Gutiérrez Rodilla (2016, p. 117), para conocer "la historia de los conceptos que maneja la ciencia, la historia de su discurso".

El análisis de esta terminología sirve, igualmente, para completar la historia del léxico español y nos descubre que la neología semántica resulta el procedimiento neológico básico de la ciencia en todas sus etapas, “porque es el más simple, el más fácil y entronca además con un proceso intrínseco al pensamiento científico, cual es la explicación [...] para ilustrar los razonamientos" (Gutiérrez Rodilla, 2013, p. 71). La analogía sobre la base de rasgos similares por la forma o apariencia es un mecanismo sumamente importante para la transmisión del conocimiento y subyace en la acuñación de algunos términos matemáticos que examinamos seguidamente y para cuya historia resultan decisivos los textos constitutivos del corpus confeccionado en el marco del proyecto de investigación Corpus para el estudio de la lengua española cientifica y matemática del siglo XVII, financiado por la Fundación Séneca.

De este modo, por ejemplo, en esta obra de Juan Carlos della Faille se relaciona entre las figuras, sin ofrecer datos adicionales, arbelo, término que Autoridades (s. v. arbelo) define como: «Figura curvilínea triangular formada de tres arcos de círculos, que cada uno de ellos es la quarta parte de la circunferencia, y hace tres ángulos agudos. Es voz de la Geometría. Tosc. tom.1. fol. 351». Igualmente, se lematiza en la obra lexicográfica de Terreros, pero también se incluye -como término ya anticuado- en los diccionarios decimonónicos de Domínguez (1853), Gaspar y Roig (1853) y Zerolo (1933) (apud NTLLE, Real Academia Española, 2001). Por último, la Academia lo recupera para su Diccionario histórico (1933). Por lo que respecta a su registro documental en los corpus diacrónicos académicos, aparece en las papeletas léxicas y lexicográficas recopiladas por la RAE en la digitalización del Fichero General en los Elementos de matemáticas de 
Lista, así como en el Compendio matemático de Tosca (Instituto de Investigación Rafael Lapesa, 2009).

En un texto coetáneo a la Geometría de Juan Carlos della Faille hallamos el registro de la voz: se trata del manuscrito Geometría práctica aplicada a la fortificación y arte militar, de autor anónimo y que ha sido fechado hacia 1660 en Sánchez Martín (2018). En esta obra es habitual que su autor inserte observaciones conceptuales y terminológicas en el curso de la resolución de los problemas; en esta ocasión, con motivo del origen etimológico del término pelecoides: «Es de notar que los geómetras griegos llamaron a estas figuras pelecoides, que significa en griego 'hacha de armas', representando a la figura de hierro que tenían antiguamente las hachas de armas para herir a los enemigos, y de las cuales hachas unas avía dobles y otras simples» ${ }^{5}$.

Al proseguir con el desarrollo geométrico incorpora otra anotación conceptual, esta vez en relación a la voz arbelo, por la semejanza formal del área de esta figura plana con la de la figura que conforma la curva pelecoide:

Podríamos servirnos del artificio para medir estas hachas dobles o simples, del cual hemos hablado en el problema 118 para medir la área de los arbelos, porque también la hacha simple de armas se puede dezir arbelo, y el arbelo se puede llamar pelecoides simple, pero como aquel artificio es más largo, daré aquí por nuestro intento otro más breve. (Anónimo, ¿1660?, fol. 44r).

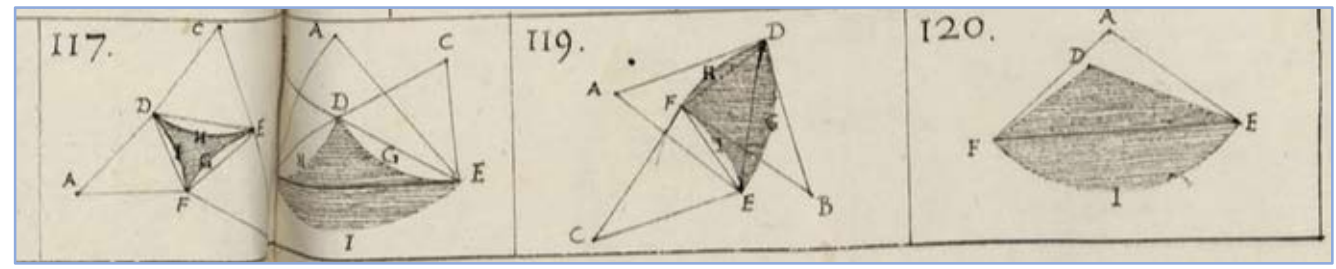

IMAGEN 1. Cálculo del área de los triángulos curvilíneos o arbelos (Geometría práctica aplicada a la fortificación y arte militar)

El matemático anónimo incorpora al final del problema 118 su observación etimológica para marcar la correspondencia terminológica entre los triángulos curvilíneos y arbelos: «Es de notar que los tales triángulos curvilíneos d'este problema se denominaron por los geómetras griegos por una palabra arbelos, que significa la figura

\footnotetext{
${ }^{5}$ Es necesario subrayar la gran deuda de la terminología matemática con la lengua y cultura griegas, visible desde el primer momento en el legado, como recuerda Etayo Miqueo (2003), de las denominaciones de las grandes ramas: aritmética, geometría y matemática.
} 
del cuchillo de los çapateros con el cual cortan el corambre, y se usa en muchas provincias de Europa» (Anónimo, ¿1660?, fol. 44r)

Efectivamente, se constata que ambas denominaciones responden a la neología semántica. La voz pelecoide, que no registra en su obra Juan Carlos della Faille, se encuentra lematizada en el diccionario de Terreros y Pando, quien la define como «Figura de Jeometría compuesta de un semicírculo, y de dos cuadrantes opuestos por su parte convexa al semicírculo, sobsteniéndole con ella, partiéndole en dos porciones iguales, y formando como una hacha. Algunos dicen Pelicoides» (Terreros y Pando, 1788: s.v.). El diccionario del lexicógrafo jesuita integra un numeroso grupo de voces matemáticas, caracterizadas todas ellas por su antigüedad y difusión, según fue anotado por Sánchez Martín (2010, p. 164-166) ${ }^{7}$. Dicho término figura recopilado más tarde por Picatoste (1862) en su Vocabulario matemático: «Pelecoide (del gr. pelekus, hacha, y eidos, forma). Figura curvilínea semejante al hacha, y formada por una semicircunferencia y dos cuadrantes en posición opuesta» ${ }^{8}$.

\footnotetext{
${ }^{6}$ Dicho significado se explica en la bibliografía especializada: "Sea un semicírculo de diámetro $A C$ y en este un punto interior $B$; si se trazan los semicírculos $A B$ y $B C$, el recinto bordeado por los tres semicírculos que Arquímedes designa con el nombre de arbelos (lezna de zapatero) es equivalente al círculo de diámetro la semicuerda $B D$, perteneciente a la tangente común a los dos semicírculos anteriores. Arquímedes agrega algunas propiedades, en especial relativas a los círculos del interior del arbelos y tangentes a sus bordes, figuras que serán estudiadas más adelante por Pappus" (Rey Pastor y Babini, 1984, p. 107).

${ }^{7}$ En este trabajo (Sánchez Martín, 2010, p. 168) se confirma que el término se admitió en su sentido matemático en los diccionarios de Domínguez (1853) y Zerolo (1895).

${ }^{8}$ A inicios del siglo XX, se halla en una relación léxica de palabras de dudosa expresión, significación u ortografía usadas en las ciencias, artes y oficios de la Construcción de la publicación El Constructor. Revista mensual de vulgarización técnica (Barcelona, febrero de 1927, número 40), que recopila la Hemeroteca Digital de la BNE: «Pelecoide. Es el rectángulo en que uno de los lados está substituido por una semicircunferencia» (El Constructor. Revista mensual de vulgarización técnica, 1927).
} 


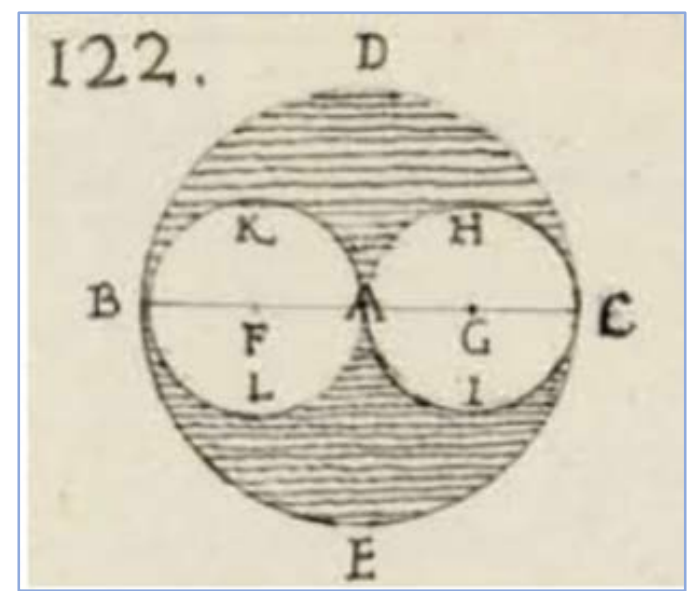

IMAGEN 2. Pelecoides (Geometría práctica aplicada a la fortificación y arte militar)

Estas dos voces específicas del registro matemático, que poseen documentación lexicográfica, sin embargo, carecen de registro documental en los corpus diacrónicos académicos, salvo en las papeletas léxicas y lexicográficas recopiladas por la Real Academia Española en la digitalización del Fichero General: por un lado, arbelo se data en los Elementos de matemáticas de Lista, así como en el Compendio matemático de Tosca, donde autorizaba la voz el primer repertorio académico; pelecoides, por su parte, se documenta en 1952, Diccionario Enciclopédico UTEHA.

Otro término formado mediante este procedimiento neológico es securícula 'en forma de arco', sentido que se obtiene de la lectura de la obra del jesuita Pauli Guldini, De centro gravitatis. Trium specierum quantitatis continuae. Liber primus, De centri gravitatis inventione (Viennae, 1635): Diversa autem a diversis auctoribus sortiuntur nomina, maxime ab iis qui sibi in diversis complacent vocabulis \& eruditis, nos eas quae tribus tantum comprehenduntur arcubus, antiquis in diversis linguis usitato vocabulo, securiculas an securiclas, quae pluribus arcubus constant. Figuras arcuatas dicimus. No hallamos ningún registro documental de la voz ni tampoco definición del término por parte de Juan Carlos della Faille, salvo su mera mención al lado de otros términos agrupados bajo la consideración de sujetos geométricos (véase en la relación léxica proporcionada más adelante). 


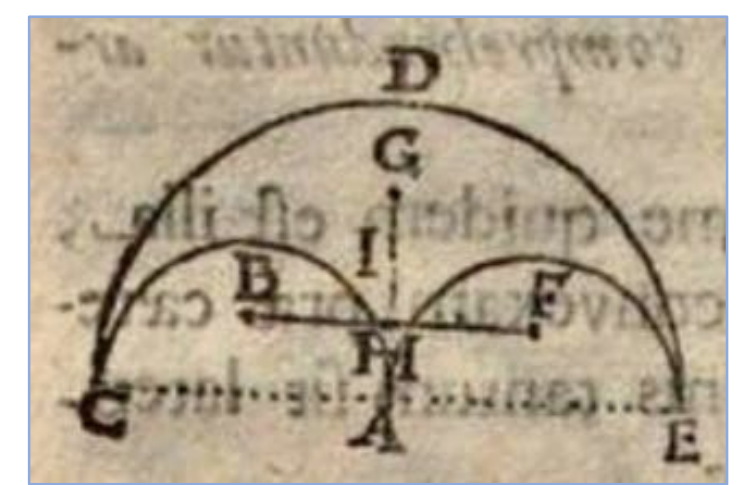

IMAGEN 3. Pauli Guldini, De centro gravitatis, 1635, pág. 88

También proporcionatriz, que es neologismo acuñado por Juan Bautista de Villalpando para referirse a una 'curva bioval'. La forma latina se localiza en la obra de Claudio Ricardo, Euclidis Elementorum Geometricorum Libros Tredecim (Antuerpiae, 1645) y, posteriormente, en el siglo XVIII se registra en un texto sobre la Refutación del célebre problema de la duplicación del cubo (Madrid, 1763): “También por las otras curbas lo resuelven los autores como Diocles por su Cissoide; Nicomedes por su conchoide; Dinostrates por la quadratiz; el padre Villalpando por la proporcionatriæ, etc.". De la proporcionatriz sí indica algunas propiedades Juan Carlos della Faille en el siguiente pasaje de su obra: "En 8 lugar, las líneas que tienen asímptotos se dividen en las que tienen dos asímptotos y en las que tienen uno solo. Dos asímptotos tiene las hipérboles y cuadratrices, uno solo las conquilis y la proporcionatriz" (Della Faille, 1640, fol. 65v).

A partir de la analogía con otra curva, conchyle 'concha, el pescado mesmo' (Nebrija, 1495), se genera la denominación de esta línea curva atribuida a Nicomedes, según anota Juan Carlos de la Faille en su obra y atestiguan en sus definiciones algunos repertorios", como el del jesuita Terreros (1786-1793): “Concoide o conquile, término de geometría, cierta figura, que inventó Nicomedes”, y lo reconocen las historias de la matemática (apud Rey Pastor y Babini, 1984, p. 120).

[Se dividen las líneas curbas en] las que mudan la concabidad como la conquilis de Nicomedes que, desde su bértex hasta una cierta distancia, tiene su concabidad hacia la línea recta con la cual nunca concurre y después la muda, y tiene su concabidad acia la otra parte. (Della Faille, 1640, fol. 64r).

\footnotetext{
${ }^{9}$ Diccionario nuevo de las dos lenguas española e inglesa de Tomás Conelly (1798): "Concoyde o conquíle. (Geom.) Cierta figura que inventó Nicomedes".
} 
Haviéndose examinado la línea curba, según el método del capítulo pasado, y constando por demostración que no es círculo, ni sectión cónica, ni cuadratriz, ni conquilis, ni la espiral de Arquímedes, que son las de las cuales asta aora tenemos alguna noticia, será menester pasar a otro examen de su naturaleça. (Della Faille, 1640, fol. 77v).

De esta tipología de líneas curvas se ocupó Caramuel, de acuerdo con el análisis proporcionado por Garma Pons:

Caramuel incorpora la definición de las líneas "conchili", líneas que sin ser equidistantes no se cortan por mucho que se prolonguen. El argumento dado por Caramuel, que lo toma del P. Mersenne, demostrando la existencia de tales líneas, en el plano, es falso, pero la idea de líneas asintóticas es válida, así como las consideraciones sobre otras superficies distintas del plano. El que las dos líneas que dibuja Caramuel no se corten implica que el triángulo que se formaría es de área infinita (Garma Pons, 2000, p. 132).

2.1. Consideraciones sobre el lenguaje como parte de la metodología CIENTÍFICA

El lenguaje conforma parte de la metodología científica, pero dicho lenguaje especializado exige de un rigor paralelo al papel desempeñado por la conceptualización en el desarrollo de la ciencia (apud Gutiérrez Rodilla, 1998, p. 24). En este sentido, la tarea definitoria adoptada por el matemático amberino en su obra está ligada a las metas propuestas: primero, el deseo de lograr la precisión terminológica, y, segundo, el afán por facilitar y divulgar los contenidos especializados que traslada, lo que va parejo a la exigencia de precisión conceptual.

En el capítulo 9 (De la posición) el matemático explica que todas las proposiciones geométricas se reducen a "raçón, proporción, potencia y posición. Las tres primeras son comunes a la jeometría y a la arismética por que assí las cantidades discretas como continuas tienen raçón, proporción y potencia” (Della Faille, 1640, fol. 40r). Sobre la última de ella, la posición, sostiene que "de la posición nace la mayor parte de los casos de las proporciones, assí en la jeometría como en las ciencias subalternadas a ella" (Della Faille, 1640, fol. 40v), pero avisa: “Aquí no se á de entender por posición el orden que tienen entre sí dos o más cantidades, como ser primera, segunda, etc., aunque los números, mayormente en la materia de las convinaciones, que conviene también a los números, impropriamente ussamos d'esta palabra de posición" (Della Faille, 1640, fol. 41r). 
En efecto, el sentido del término corresponde más bien a 'suposición' como define Autoridades: "Y assí se dice en la Arithmética, La regla de falsa posición. Latín. Positio.". En la aritmética distingue Della Faille estas tres reglas: "a saver la de tres, de falsa posición y de aligación; y en el áljebra no hay más que una. Y todas bien miradas no son sino la regla de tres multiplicada" (Della Faille, 1640, fol. 95v) ${ }^{10}$.

\footnotetext{
Y se puede este modo de solución llamar por falsa posición y Euclides se bale de él (en el libro 4) para describir un pentágono dentro de un círculo. (96v).

Lo mismo se vee en la proposición de el tercero adonde, desde un puncto dado fuera de el círculo, saca una tanjente a él; y en el 6 , adonde divide la línea recta en partes iguales; y en otros problemas, cuyas soluciones tienen algo de la regla de falsa posición; y este modo se declarará con diferentes exemplos en su lugar por ser de tanto probecho. (97r).
}

Utilizada por los antiguos, gozó de una gran popularidad en los textos matemáticos del siglo XVI para resolver algunos problemas, y así se documenta en los textos del DICTER, repertorio que la define como 'La que enseña a resolver algunos problemas por medio de un número supuesto falso, del que se deduce el valor de la incógnita tras compararlo con los datos del problema”. (DICTER, Mancho Duque, 2010-2017).

Corresponde a los científicos velar por el uso correcto de la terminología, aspecto ya tratado por los estudios sobre la historia de la lengua y de la ciencia españolas. En nuestro ámbito, ya ha sido referida la elevada conciencia terminológica de los ingenieros españoles observada desde finales del XVI y durante la centuria siguiente. Recuérdense a este propósito las críticas de Cristóbal de Rojas sobre el desconocimiento de la terminología en el ámbito de la fortificación, según Sánchez Martín, 2013, p. 149-152), o, tal como se ejemplifica en Sánchez Martín (en prensa), la preocupación terminológica visible en el tratado Escuela de Palas (1693) sobre los nombres del revellin y media luna, que designan sendas obras de fortificación. Tal sucede con el matemático antuerpiense, según se ha podido comprobar en el apartado anterior del presente estudio cuando se ejemplificaron los mecanismos de reformulación en tanto que procedimiento característico del discurso científico para clasificar y definir los conceptos.

10 “Aligación. Liga, mezcla o composición de diferentes especies, de que resulta otra especie media. Lat. Alligatio, onis. TOSC. tom. 1. Regla de aligación se llama en la Arithmética aquella con que se resuelven las qüestiones tocantes a ella" (Autoridades).

RILEX. VOLUMEN MONOGRÁFico

ANÁLISIS TERMINOLÓGICO DEL MANUAL GEOMÉTRICO (MADRID, 1640) DE JUAN CARLOS DELLA FAILLE 
En el capítulo primero, por medio de el predicamento de la cantidad, hemos dado noticia de los subjetos y predicados de la jeometría para que se conozcan los términos, pues, según el horden de la dialéctica, cuyo oficio es governar y dirijir las tres operaciones de nuestro entendimiento, primero se á de formar la aprehensión simple con darlas las especies y conceptos simples, de las cuales se han de formar las proposiciones que pertenecen a la segunda operación, que se llama el juicio, para pasar últimamente a la tercera operación, que consiste en el discurso sacando de proposiciones o conocidas por sí mismas o antes demostradas otras no conocidas. (Della Faille, 1640, fol. 10v).

Apagogé se llama el passo de una proposición a otra la cual, cuando fuere conocida, será también conocida la otra. [...] La cual translación se llama apogogé en griego y aductión en latín, y trata de ella Aristóteles en el libro primero de los Priores, adonde define diferentes especies de aductiones, pero la que hace a nuestro propósito es la segunda, que dice abductio est cum pauca media intercendens interpostremum et medium ita enim fin ut ad cognitionem proprius umnino accedamus. Assí los traducen los intérpretes de griego en latín, pero más claramente y más conforme a la naturaleça de la abductión si dijieren cum pauciota media intercidunt inter postremum et medium, porque para declarar la esencia de el apagogé se á de notar que algunas proposiciones están muy remotas de los principios o de las proposiciones conocidas; y por esto para demostrarlas es menester muchas entremedias entre ellas y los principios conocidos. (Della Faille, 1640, fol. 97r-98r) ${ }^{11}$.

Además de por ser elementos ineludibles de la metodología científica en tanto que partes constituyentes, -como demostró Gutiérrez Rodilla (1998, p. 24, 2013, p. 69)entre el lenguaje y los conceptos de la ciencia, y, por ende, su dominio, se establece una relación de necesidad. Sirvan estas palabras del matemático antuerpiense para ilustrarlo: "La noticia de la abductión es de suma importancia en la jeometría y en todos las esciencias matemáticas y lo mismo será en los demás, porque pierde mucho tiempo y trabajan sin provecho los que la ignoran" (Della Faille, 1640, fol. 99r).

La intención por parte de Juan Carlos della Faille de divulgar estos contenidos temáticos en español se acompaña de la conveniente necesidad de cumplir con los propósitos de precisión terminológica, aspecto ampliamente reconocido por los científicos españoles de la centuria anterior cuando recomendaban a sus lectores, principalmente técnicos, explícitamente en sus obras la necesidad de manejar el vocabulario matemático como vía para acceder a los conocimientos ${ }^{12}$. El manual

11 “Abducción. Especie de argumento cuyo término mayor está evidentemente contenido en el medio y en que este no está íntimamente ligado al menor. (Domínguez, Suplemento 1869 s.v. abducción). Aligación. Liga, mezcla o composición de diferentes especies, de que resulta otra especie media. Lat. Alligatio, onis. TOSC. tom. 1. Regla de aligación se llama en la Arithmética aquella con que se resuelven las qüestiones tocantes a ella" (Autoridades). Demostración de la verdad de una proposición, por lo absurdo de la contraria, probándolo. (Domínguez, 1853, s.v. apagogía).

${ }^{12}$ Esta voluntad, también, explicó la eclosión de repertorios y glosarios renacentistas elaborados en castellano con la pretensión de aclarar el sentido de los tecnicismos insertos en los textos científicos y 
Método de la geometría supone un testimonio directo de una obra confeccionada en español fruto de la actividad matemática desarrollada por Juan Carlos della Faille en los Reales Estudios del Colegio Imperial madrileño. Igualmente representa el bagaje acumulado resultado de su actividad de estudio particularmente centrado en las cuestiones sobre geometría, así como de las ocupaciones docentes y académicas que desempeñó en nuestro país.

A propósito del concepto de demostración, en el capítulo que le dedica, advierte:

Berdad es que si examinaran los discursos y demostraciones de muchos autores, que se hallarían muchas d'estas, por estar tan poco hordenadas las esciencias y reducidas a el horden natural de propriedad y de posteridad, causalidad y dependencia que tienen unos predicado de otros, pero aquí no pretendo repreender a nadie, antes alavar y estimar en mucho los travajos de todos, pues han procurado adelantar las esciencias. (Della Faille, 1640, fol. 22r-22v).

Antes de sostener taxativamente: "La mayor falta que tenemos es el no comprehender bien los términos" (Della Faille, 1640, fol. 22v). Para suplir estas carencias, recomienda la útil tarea de la recopilación léxica: "Se á de procurar de comprehender bien la significación de cada término y para esto será de gran probecho tener la difinición o descripción o la declaración de cada uno de ellos y será trabajo utilíssimo recojer de todos los autores las difiniciones que tienen y definir, descrivir o explicar los términos que no se hallaren explicados en los autores" (Della Faille, 1640, fol. 27v-28r).

Ahora bien, el objetivo de su obra es instruir al que estudiare-como afirma-, de ahí que para facilitar dicha instrucción reúna bajo el capítulo quinto (De la imbención y multiplicación de los términos jeométricos) la recopilación y clasificación conceptual de los términos privativos de esta ciencia geométrica, "en la cual no se admite cosa ninguna que no sea científica en rigor" (Della Faille, 1640, fol. 17v), como sentencia el matemático.

El jesuita aconseja, así, como primera empresa para su estudio la tarea de la documentación terminológica:

técnicos. Recuérdense, además, con Sánchez Martín (2013, p. 149), las obligaciones docentes de los titulares de la cátedra, como la de matemáticas y fortificación, instituida en la corte madrileña con la misión de enseñar en la corte matemáticas, geometría y fortificación, consistentes en explicar los conceptos matemáticos, enseñar el manejo de los instrumentos geométricos y leer otras materias especializadas (también Vicente Maroto y Esteban Piñeiro, 2006, p. 178-180). 
Disputamos en los prolegómenos de la dialéctica si hay arte en el mundo que enseñe a hallar términos nuebos en alguna facultad y, pues la lójica govierna la primera aprehensión, si ella tiene por oficio hallarlos y imprimirlos en el entendimiento o enseña solamente ussar bien de los que tenemos, bien cierto es que para començar hemos de tener algunos y después no falta arte de multiplicarlos. Y, assí, el que quisiere començar el estudio de la jeometría tome un cartapacio y recoja en él todos los términos y bocablos proprios que hallare, informándose de quién le puede dar noticia de ellos y biendo los libros de los jeómetras. (Della Faille, 1640, fol. 27r-27v).

Se confirma de este modo la asunción de que la terminología es la columna vertebral de la ciencia:

Supuesto el dicho índice, se á de procurar de comprehender bien la significación de cada término y para esto será de gran probecho tener la difinición o descripción o la declaración de cada uno de ellos y será trabajo utilíssimo recojer de todos los autores las difiniciones que tienen y definir, descrivir o explicar los términos que no se hallaren explicados en los autores (Della Faille, 1640, fol. $27 \mathrm{v}-28 \mathrm{r})$.

El matemático procede a la división de estos tecnicismos $y$, por ende, a su clasificación ${ }^{13}$, de acuerdo con la concepción de la dialéctica, 'el arte de discutir, conversar', en tanto que "arte de dividir las cosas en género y especies, o clasificar los conceptos, para poder someterlas a examen y discusión" (Quintás Alonso 2002: s. $v$. dialéctica ${ }^{14}$. Su afán pedagógico justifica la decisión de enumerarlos: "Y, aunque el intento d'este tratado sea instruir a el que estudiare y no poner por extenso todas las cossas que se mandarán hacer, con todo esto, para mayor perfección de él y para mayor comodidad y haorro de tiempo, se ará esta colección, assí de los términos reducidos a orden alfabético (Della Faille, 1640, fol. 28r).

\footnotetext{
13 Siguiendo a Velarde Lombaña (1989), en la ciencia aristotélica aparece el ideal de clasificación. "El silogismo supone una clasificación de los seres, y no vale más que en virtud de dicha clasificación, tanto si se interpreta desde el punto de vista intensional como desde el punto de vista de la extensión. Para Aristóteles, lo mismo que para Platón, definir es clasificar" (1989, p. 25).

${ }^{14}$ La teoría fundamental de la dialéctica es una teoría de la argumentación (apud Flórez Miguel, 2001, p. 41), diferente de la teoría fundamental de la matemática, un saber "que no versa sobre argumentos ni comentarios de otros libros, sino de un saber que se dirige a las cosas mismas intentando descubrir el orden de las cosas. [...] El procedimiento de las matemáticas no mira a la persuasión como es el caso de la retórica y la lógica, sino a la invención. Y para la invención y el descubrimiento el paradigma fundamental es la matemática, que no persigue un procedimiento argumentador, sino constructivo" (Flórez Miguel, 2001, p. 42).
} 
Así, agrupa estos términos y grupos designativos concernientes a las matemáticas según su consideración como sujetos ${ }^{15}$, predicados ${ }^{16}$ ("partes y otras cossas en orden a los subjetos"), operaciones y pasiones ${ }^{17}$.

- Subjetos son punto, cantidad contina, línea, superficie, cuerpo, línea recta; triángulo rectilíneo, equilátero, isósceles, escaleno, rectángulo, obtusángulo, acutángulo; paralelogramo cuadrado, rectángulo, prolongado, rombo, romboide, trapecio, polígono; figura regular, figura irregular; ángulo rectilíneo, agudo, obtusso, recto, ángulo curbilíneo, mixtilíneo, de contingencia; círculo, sector, secmento, lúnula, arbelo, securícula, elipsis, ipérbola, parábola, cuadratriz, conquilis, espirales, esfera, esferoides, conoides, parabólico, conoides, hiperbólico, cilindro, cono, paralelopípedo, prisma, pirámides, cubo, octaedro, dodecaedro, icosaedro, cuerpo regular, cuerpo irregular. (Della Faille, 1640, fol. 30r).

- Partes y otras cossas en orden a los subjetos son centro, polos, vértex, puncto de intersectión, puncto de el contacto, punto de el concurso, diámetro, cuerda, subtensa, tanjente, secante, diámetros conjugatos, asis, lado, basis, altura o perpendículo, secmentos de la bassis; ángulo plano, tanjente, plano, secante, sectión común, lado recto, lado transverso, figura inscripta, figura circunscripta; paralelo, perpendicular, obliquo, asímptoto, ordinatín aplicada, máxima, igual, mínima. (Della Faille, 1640, fol. 30r-30v).

- Operaciones son descrivir, producir, aumentar, disminuir, multiplicar, dividir, transmutar, cortar, tocar, aplicar, inclinar, medir. (Della Faille, 1640, fol. 30v).

- Pasiones ${ }^{18}$ más unibersales son la composición, la descriptión, la posición, la comparación, la igualdad, el excesso y el defecto, el agregado, la diferencia, la aumentación, la diminución, la multiplicación, la división, la aplicación, la transmutación, el concurso, la sectión, el contacto, la congruencia, la equidistancia, la inclinación, la raçón de asíntotos o no, concurrencia, la

15 "Se dice que la materia que es sujeto en tanto que soporta la forma, o bien se dice que la substancia es sujeto en tanto que soporta los accidentes" (Quintás Alonso, 2002, p. s. v. sujeto).

16 "Lo que se afirma o niega de un sujeto. Si consideramos las cuatro clases de proposiciones, en la Lógica tradicional: todo $\mathrm{S}$ es $\mathrm{P}$, ningún $\mathrm{S}$ es $\mathrm{P}$, algún $\mathrm{S}$ es $\mathrm{P}$, algún $\mathrm{S}$ no es $\mathrm{P}, \mathrm{S}$ denota al sujeto, $\mathrm{P}$ al predicado". (Quintás Alonso, 2002, s. v. predicado).

"Aristóteles en Los Tópicos se refiere a los distintos modos de relación sujeto/predicado, articulados sobre los criterios de convertible/no convertible y esencial/no esencial; estamos ante los diversos modos de relacionar un predicado con un sujeto. Si la relación sujeto predicado es convertible y esencial se denomina definición; si es convertible y no esencial, propiedad; si es esencial y no convertible, género o diferencia; si no esencial y no convertible, accidente. Porfirio (Isagoge I) presentó cinco predicables: el género, la especie, la diferencia, lo propio y el accidente". (Quintás Alonso, 2002: s. v. predicable).

17. "Pasión 'que es sufrido, lo que es soportado'. Aristóteles usa el término para referirse a la afección o modificación padecida en mayor o menor grado por algo ["es padecer: ser cortado, ser quemado"]" (Quintás Alonso, 2002: s. v. pasión).

${ }^{18}$ Más adelante, precisa: "Las pasiones proprias de la posición son las que se siguen en los punctos estar en derecho o no en derecho, estar dentro o fuera o en el extremo en las líneas y superficies, también se halla estar en derecho o no estar en derecho y en ajustarse una con otro por medio de la superposición en líneas finitas, ángulos y figuras -siendo iguales y semejantes-, ser paralelos, perpendiculares, oblicuos, cortarse, tocarse, concurrir, acer ángulo, inscribir, circunscribir, etc." (Della Faille, 1640, fol. 40v). 
dimensión, la semejança, la desemejança, la conmensurabilidad, la inconmensurabilidad, la raçón, la proporción, la potencia plana, la potencia sólida. (Della Faille, 1640, fol. 30v-31r).

Responde tal diferenciación a la lógica aristotélica: "La diferencia es suficiente para poner en orden el conjunto de los objetos, es decir, para clasificarlos y, por consiguiente, para definirlos, ya que la diferencia contiene en sí misma el género" (Velarde Lombaña, 1989, p. 28). Por consiguiente, además de estos tecnicismos matemáticos, y en consonancia con este ideal de clasificación, aparecen en esta obra definidos otros vocablos propios de la lógica, por ejemplo, categoría, especie, género, juicio, medio, pasión, predicado, propiedad, propio, razón, repugnar, repugnante o términos, entre otros. Como puede cotejarse en Sánchez Martín (en prensa), todos ellos han sido incluidos en la recopilación léxica confeccionada y definidos junto con los restantes tecnicismos empleados por el jesuita en su Método de la geometría.

La actividad científica de este jesuita, como la de muchos otros de la Compañía, estuvo relacionada con la enseñanza. "El curso normal de la formación de la Compañía de Jesús incluía el estudio de la física, generación y corrupción, meteorología y el cielo de Aristóteles". Las clases servían de introducción a la filosofía natural de Aristóteles, si bien, al no contar con un texto oficial se ofrecía -según O’Neill y Domínguez, 2001, s. v. ciencias físicas - "la ventaja de mantener las clases abiertas a la admisión de nuevos descubrimientos". "Los comentarios jesuíticos sobre Aristóteles abundaron especialmente en el siglo XVII, centuria, como la anterior, en que las materias hoy llamadas ciencias se enseñaban, según el esquema aristotélico, en los cursos de filosofía natural y matemáticas” (apud O’Neill y Domínguez, 2001, s.v. ciencias naturales y matemáticas). Ahora bien, otros comentaristas opinaban que Aristóteles debía dejarse de lado cuando sus teorías no estaban conformes con los hechos:

Esa posición supuso un paso fundamental al rechazar la autoridad tradicional en física y fomentar la aceptación de nuevas hipótesis, tal como la existencia de corpúsculos o átomos. [...] Antes de 1650 los jesuitas estaban divididos en cuanto a la defensa de la antigua visión geocéntrica del universo. Unos la ponían abiertamente en duda, como Grégoire de Saint Vincent. Otros se resistían a abandonarla, como Clavius. (O’Neill y Domínguez, 2001, s. v v ciencias físicas). 
La colección algunos de estos términos referidos en el capítulo quinto se reproduce en el sexto (De el modo con que los subjetos jeométricos se han de pasar por los predicados y por los demás términos):

Para que esto se aga con más facilidad y compendio, de suerte que de una bista se comprenda todo, lo mejor será rayar un pliego a modo de cuadrícula dejando a la mano izquierda lugar para escribir todos los subjetos y arriba también para escribir los predicados y operaciones jeométricas; y, en el cuadrado que está en el concurso común, hacer una señal que declare que el predicado conviene a el subjeto o no, como se ve en una figura que está aquí. (Della Faille, 1640, fol. 31v)

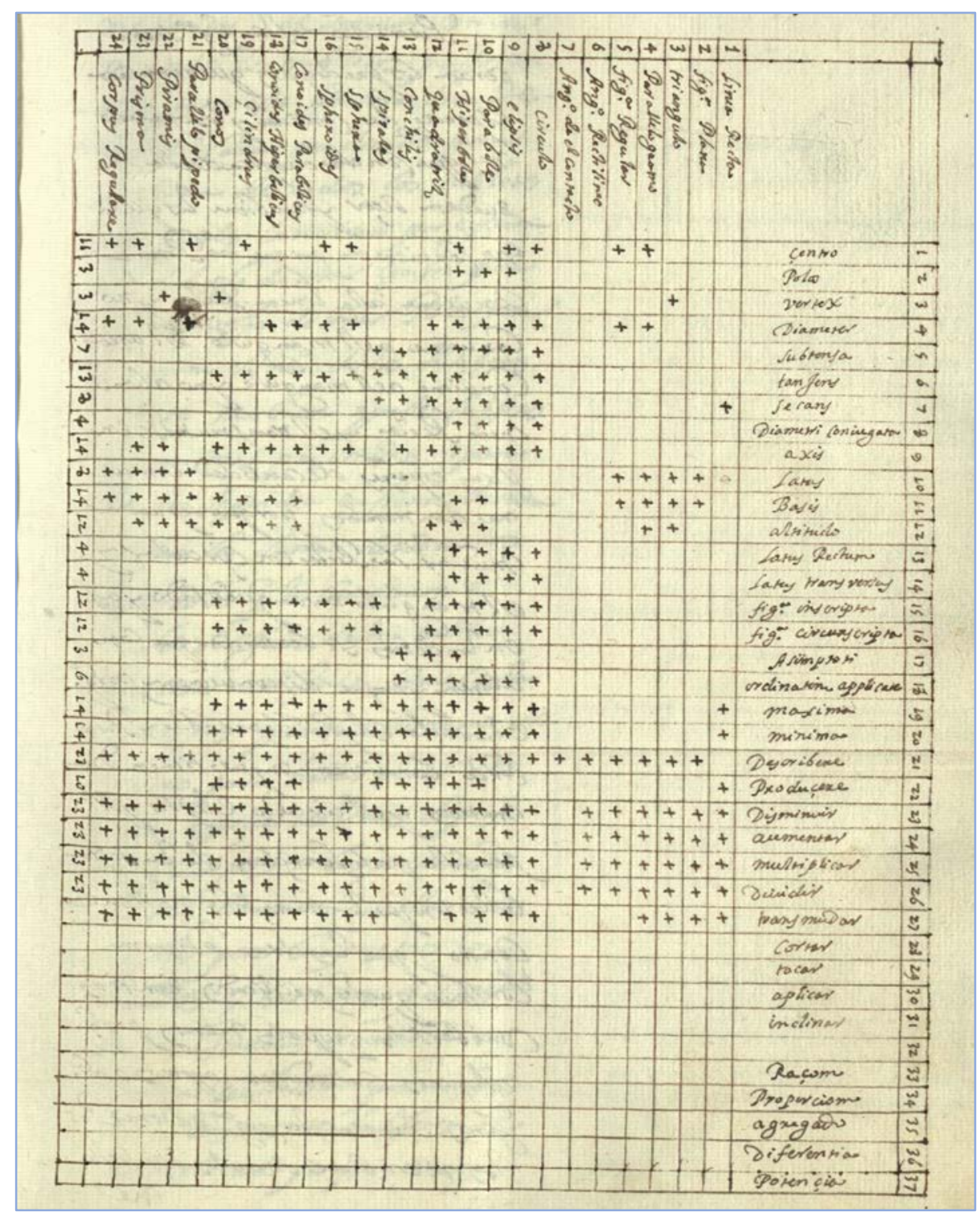

Imagen 4. Tabla de correspondencias entre las propiedades geométricas (Della Faille, 1640, fol. $32 \mathrm{r}$ ) 
Es uno más de los elementos que conduce a pensar en el denodado interés didáctico de la obra, propósito patente cuando alude el autor a la ordenación dada a los contenidos, a las disposiciones que adopta en la materia de las combinaciones o, por ejemplo, en las invitaciones al empleo de esquemas y dibujos para aprehender complejos conceptos, como veremos seguidamente.

Es evidente, como dijimos, que precisamos de la palabra para expresar conceptos científicos, pero a la vez la imagen permite visualizarlos y comprenderlos, como observa el jesuita antuerpiense:

[Capítulo quinto. De la imbención y multiplicación de los términos jeométricos] Y pues las cossas matemáticas no dependen solamente de voces y palabras, sino también de las figuras sin las cuales no se hace concepto de ellos, será necesario en otro papel descrivir todas las figuras con buen dibujo [...] porque es increíble el alibio que da a la imajinatiba el ver delante de sí lo que se á de representar (Della Faille, 1640, fol. 28v-29r).

[Capítulo 19. Del modo de examinar las líneas curbas halladas para descubrir de qué especie son] Para ayudar a la mecánica en esta parte será bien tener un cartapacio en que estén descriptas, con mucha puntualidad, todas las especies de las líneas curbas que tubiéremos para que la misma vista de ellas, y el modo de su inflexión y/o curbidad, se imprima poco a poco en la imajinación, y que con sola la vista aprendamos a conocerlas y distinguirlas unas de las otras. $Y$ este cartapacio se pondrá también en el apéndix de este tratado. (Della Faille, 1640, fol. 77r).

Es por ello que los esquemas y los dibujos han servido para comprender y transmitir conceptos complejos, o han ayudado a priorizar la información. Podemos decir que el matemático piensa de manera pictórica y, asimismo, argumentar que el recurso a las imágenes visuales se emplea para auxiliar el alto nivel de abstracción de los conceptos que trata, de ahí que, en diversos pasajes de su obra, el matemático jesuita recomiende la apoyatura de las imágenes y la reproducción material de las figuras geométricas:

[Capítulo 24. De el modo de hallar y demonstrar teoremas] Cuando se buscare la demonstración de algún teorema de cuya verdad no nos consta, podremos, en primer lugar, valernos de la figura bien echa y en diferentes maneras porque, si fuere falsa en alguna de ellas, aun mecánicamente se descubrirá la falsedad. Procedérase después a otro examen por números, guardando los mismos preceptos que hemos dado. (Della Faille, 1640, fol. 108v).

El primer exercicio, y el más fácil de todos, es para el que comiença a estudiar matemáticas: es hacer figuras de todos jéneros porque, además de que el hacerlas bien y punctuales es muy necesario, se imprime en la imajinación la propria especie de cada una, de suerte que algunos tienen tanta destreça que, dada cualquiera línea curba, a poco más o menos juzgan si es pedaço de círculo, de elipsis, hipérbole, parábole, etc. Y es de mucho probecho cuando acasso caemos en algún lugar jeométrico para descubrir la especie; y, assí, en primer lugar, se han de describir las sectiones cónicas muchas veces, las espirales, conquilis, cuadratriz, etc. (para que se imprima su figura en la imajinación), su centro, polos, asímptotos, diámetros conjugados, ordinatín aplicadas, tanjentes, etc., 
porque, cuando especulamos de alguna materia, las podamos descrivir con la imajinación sola y ver adónde tocan o cortan, etc. (Della Faille, 1640, fol. 116r-116v).

Las instrucciones sobre la organización de este material gráfico (Y este cartapacio se pondrá también en el apéndix de este tratado) o la preocupación del jesuita por los materiales utilizados para realizar los dibujos técnicos sirve para justificar el propósito didáctico de esta obra:

Para que este exercicio se haga con más probecho, será bien hacer las figuras en medios pliegos o pliegos enteros para que sean grandes y despejados y se puedan guardar. Y se tomará otro papel y cartapacio para apuntar lo que se ofreciere, dando a cada pliego y figura su número, por exemplo, a la figura 4 de el folio 7, etc., porque las mismas figuras pueden servir otras veces y, bolviendo a mirar las mismas, se nos ofrecerán cossas que la primera y segunda y tercera vez no se descubrían. (Della Faille, 1640, fol. 118r-118v).

Dibujar implica análisis, comprensión y práctica, coleccionar los conocimientos, al tiempo que esta práctica a partir de los contenidos es, por excelencia, un ejemplo del carácter pedagógico que posee este tratado:

[Capítulo 27. Varios exercicios para los que estudian jeometría] Aunque por las reglas y preceptos susodichos se alcance la esciencia, con todo esto el exercicio es necesario para la facilidad de la práctica; y muchas veces descubre el exercicio lo que con mucha especulación no se alcança. (Della Faille, 1640, fol. 116r).

Los ejercicios geométricos propuestos al final de la obra están encaminados al dominio de esta ciencia y sirven de apoyatura para alcanzar el nivel de abstracción de los contenidos. Por ejemplo, el primer ejercicio propone la construcción de figuras diversas de forma que el lector u oyente se forme una imagen mental de las mismas y pueda operar posteriormente con la incidencia de estas figuras geométricas.

Efectivamente, recordar sobre los dibujos correctos y disponer de este material ordenado implica aprender dibujando, lo que resume claramente, a nuestro juicio, el propósito pedagógico que el autor persigue con esta obra, manual dirigido a los estudiantes de los Reales Estudios del Colegio Imperial madrileño con el propósito de mostrar las proposiciones geométricas a un nivel matemático básico. Todos estos ejemplos ponen de manifiesto nuestra aptitud para el "pensamiento simbólico, de imaginar y articular ideas que representan realidades o imaginaciones” (Sánchez Ron, 2019, p. 70). 


\section{VALORACIÓN FINAL}

Finalmente, es imposible agotar la descripción y caracterización del vocabulario matemático contando únicamente con los datos ofrecidos de entre los disponibles en este tratado. En este sentido, para un conocimiento cabal del análisis del plano léxico efectuado debe prestarse atención a los datos compilados en Glosario de voces lógicas y matemáticas y que ha sido confeccionado a partir del Método de la geometría (Sánchez Martín, 2019), cuya macroestructura está conformada, como es lógico, por una considerable cantidad de tecnicismos geométricos, así como voces adscritas a las matemáticas, al tiempo que por un número elevado de voces pertenecientes a la lógica, principalmente por la temática de los contenidos tratados por el jesuita en esta obra, esto es, las reflexiones filosóficas y las conexiones con las matemáticas.

Sin embargo, a partir del conjunto terminológico recopilado en el presente trabajo, sí pueden extraerse algunas conclusiones; así, por el contexto en el que se enmarca la redacción de este manuscrito, se puede corroborar, por un lado, la importancia del Método de la geometría en el marco de las tareas docentes asumidas por Juan Carlos della Faille y, por otro, la preocupación de este matemático por difundir los conceptos, definir el vocabulario matemático y, por consiguiente, trasladar en lengua española los contenidos especializados de que trata en su obra.

\section{BIBLIOGRAFÍA}

Durán Guardeño, A. J. (2000). De cómo se gestó y vino al mundo el cálculo infinitesimal. En A. J. Durán Guardeño, El legado de las matemáticas. De Euclides a Newton: los genios a través de sus libros. Sevilla: Universidad de Sevilla: Real Sociedad Matemática Española, pp. 226-276.

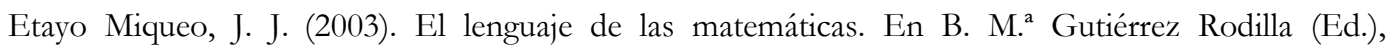
Aproximaciones al lenguaje de la ciencia. Burgos: Colección Beltenebros, pp. 345-370.

Flórez Miguel, C. (2001). Otra cara del humanismo. En M. ${ }^{a}$ J. Mancho Duque y C. Blas Nistal (Eds.), Pórtico a la ciencia y a la técnica del Renacimiento. Salamanca: Universidad de Salamanca, pp. 11-43.

Garma Pons, S. (2000). Caramuel y la revolución en las matemáticas del siglo XVII. En M.a del C. Escribano Ródenas (Coord.), Matemáticos madrileños. Madrid: Anaya, pp. 105- 140.

Gutiérrez Rodilla, B. M. (1998). La ciencia empieza en la palabra. Análisis e bistoria del lenguaje científico. Barcelona: Ediciones Península. 
Gutiérrez Rodilla, B. M. ${ }^{a}$ (2013). Sobre la formación de palabras y el léxico científico: algunas nociones generales y varias preguntas al aire, Anexos de Revista de Lexicografía, 19, pp. 69-78.

Gutiérrez Rodilla, B. M. ${ }^{a}$ (2016). Reflexiones historiográficas sobre el léxico científico y los repertorios lexicográficos, Anexos de Revista de Lexicografía, 35, pp. 117-128.

Instituto de Investigación Rafael Lapesa de la Real Academia Española (2009). Fichero General de la Lengua Española [en línea]. http://web.frl.es/fichero.html

López Piñero, J. M. a et al. (1983). Diccionario histórico de la ciencia moderna en España. Barcelona: Ediciones Península.

Mancho Duque, M. ${ }^{a}$ J. (Dir.) (2010-2017). DICTER. Diccionario de la ciencia y la técnica del Renacimiento. Salamanca: Ediciones Universidad de Salamanca [en línea]. Http://dicter.usal.es/.

Meskens, A. (2005). Joannes della Faille s.j. Mathematics, Modesty and Missed Opportunities. Bruxelles: Institut Historique Belge de Rome.

O’Neill, Ch. E., y J. M. ${ }^{a}$ Domínguez (Dirs.) (2001). Diccionario histórico de la Compañía de Jesús: biográficotemático. Madrid: Universidad Pontificia Comillas.

Picatoste y Rodríguez, F. (1862). Vocabulario matemático-etimológico. Madrid: Imprenta y Librería de D. E. Aguado.

Quintás Alonso, G (2002). Términos y usos del lenguaje filosófico. València: Universitat de València.

Real Academia Española (2001). Nuevo tesoro lexicográfico de la lengua española. Madrid: Espasa Calpe.

Rey Pastor, J. y J. Babini (1984). Historia de la matemática, Vol. 1. De la antigüedad a la Baja Edad Media. Barcelona: Gedisa.

Sánchez Martín, F. J. (2009). Estudio del léxico de la geometría aplicada a la técnica en el Renacimiento bispano. Salamanca: Universidad de Salamanca.

Sánchez Martín, F. J. (2010). La recepción de tecnicismos matemáticos en la lexicografía española decimonónica, Ianua. Revista de Philologia Romanica, 10, pp. 143-174.

Sánchez Martín, F. J. (2012). Las obras matemáticas españolas del siglo XVII: una propuesta de estudio, Diálogo de la Lengua, IV, pp. 1-23.

Sánchez Martín, F. J. (2013). La conciencia terminológica de los matemáticos e ingenieros renacentistas y su preocupación por la divulgación científica, Anuario de Estudios Filológicos, XXXVI, pp. 147164.

Sánchez Martín, F. J. (2018). Corpus especializado para el estudio de la lengua de la geometría española del siglo XVII, Philologica Canariensia, 24, pp. 113-130.

Sánchez Martín, F. J. (2019). Método de la geometría (1640) de Juan Carlos della Faille. Estudio y edición. Murcia: Universidad de Murcia, Servicio de Publicaciones (Editum Signos).

Sánchez Martín, F. J. (en prensa). Análisis del manuscrito BNE-9118 referido a las prácticas necessarias geométricas para traçar todo género de figuras, edificios y fortificaciones, y medirlas. En M. De Beni (Ed.), 
Imagen y discurso técnico-cientifico en español. Léxico, análisis discursivos, obras de especialidad. Mantova: Universitas Studiorum (Pliegos Hispánicos, n. 7).

Sánchez Ron, J. M. (2019). El valor de la Historia de la Ciencia, Cuadernos de pedagogía, 495, pp. 70-72.

Simón Díaz, J. (1992). Historia del Colegio Imperial de Madrid (Del Estudio de la villa al Instituto de San Isidro: años 1346-1955). Madrid: Instituto de Estudios Madrileños.

Terreros y Pando, E. (1786-1793). Diccionario Castellano con las voces de las Ciencias y las Artes. Madrid: Arco Libros.

Van de Vyver, Omer (1977). Jan-Karel della Faille (1597-1652), Jezuïeten, 35, pp. 247-253.

Velarde Lombaña, J. (1989). Historia de la lógica. Gijón: Servicio de Publicaciones de la Universidad de Oviedo. 